Single-molecule switching with non-contact atomic force microscopy

This article has been downloaded from IOPscience. Please scroll down to see the full text article.

2011 Nanotechnology 22245701

(http://iopscience.iop.org/0957-4484/22/24/245701)

View the table of contents for this issue, or go to the journal homepage for more

Download details:

IP Address: 134.93.197.106

The article was downloaded on 21/04/2011 at 06:12

Please note that terms and conditions apply. 


\title{
Single-molecule switching with non-contact atomic force microscopy
}

\author{
Jens Schütte ${ }^{1,4}$, Ralf Bechstein ${ }^{2,5}$, Philipp Rahe ${ }^{1}$, Heinz Langhals ${ }^{3}$, \\ Michael Rohlfing ${ }^{2}$ and Angelika Kühnle ${ }^{1}$ \\ ${ }^{1}$ Institut für Physikalische Chemie, Johannes Gutenberg-Universität Mainz, \\ Jakob-Welder-Weg 11, D-55099 Mainz, Germany \\ ${ }^{2}$ Fachbereich Physik, Universität Osnabrück, Barbarastraße 7, D-49076 Osnabrück, Germany \\ ${ }^{3}$ Department Chemie, Ludwig-Maximilians-Universität (LMU) München, \\ Butenandtstraße 13, D-81377 München, Germany \\ E-mail: jenschue@uos.de
}

Received 7 January 2011, in final form 28 February 2011

Published 20 April 2011

Online at stacks.iop.org/Nano/22/245701

\begin{abstract}
We report upon controlled switching of a single 3,4,9,10-perylene tetracarboxylic diimide derivative molecule on a rutile $\mathrm{TiO}_{2}(110)$ surface using a non-contact atomic force microscope at room temperature. After submonolayer deposition, the molecules adsorb tilted on the bridging oxygen row. Individual molecules can be manipulated by the atomic force microscope tip in a well-controlled manner. The molecules are switched from one side of the row to the other using a simple approach, taking benefit of the sample tilt and the topography of the titania substrate. From density functional theory investigations we obtain the adsorption energies of different positions of the molecule. These adsorption energies are in very good agreement with our experimental observations.
\end{abstract}

(Some figures in this article are in colour only in the electronic version)

\section{Introduction}

The invention of the scanning tunneling microscope (STM) in 1982 by Binnig et al enabled not only imaging conducting surfaces at the atomic scale but also manipulating single atoms and molecules on these surfaces [1-4]. Both imaging and manipulation with an STM are generally well understood and under excellent control. A severe disadvantage of STM, however, constitutes its limitation to conducting surfaces. Noncontact atomic force microscopy (NC-AFM), on the other hand, measures the force acting between an atomically sharp tip and the surface and can, thus, be used to image both conducting [5] and insulating surfaces [6-8] with atomic resolution. Recently, lateral and vertical manipulation of single atoms has been demonstrated using NC-AFM [9-13] and studied theoretically [14-17].

In contrast to the precise control in single-molecule manipulation using STM, the manipulation of single molecules

\footnotetext{
4 Present address: Dr. Eberl MBE-Komponenten GmbH, Gutenbergstraße 8, D-71263 Weil der Stadt, Germany.

5 Present address: Interdisciplinary Nanoscience Center (iNANO), Department of Physics and Astronomy, Aarhus University, Ny Munkegade, DK-8000 Aarhus C, Denmark.
}

using NC-AFM is still far from being mature. Several theoretical studies exist, dedicated to understanding the manipulation of single molecules using an NC-AFM tip [18-20], but up to now only a few experiments have been reported upon manipulating molecules with NC-AFM. In particular, several challenges have to be overcome when performing the manipulation of single molecules with $\mathrm{NC}-\mathrm{AFM}$ on insulating surfaces. At room temperature, the molecular surface diffusivity is usually very high, resulting in cluster formation and molecular crystal growth [21-23]. High molecular mobility hampers controlled manipulation of single molecules at room temperature and requires the experiments to be performed at low temperatures. So far, however, no lowtemperature NC-AFM manipulation experiments of a single molecule on a non-conductive surface have been reported.

Successful NC-AFM manipulation has been presented in 2006 by Hirth et al, demonstrating lateral manipulation of defects on the $\mathrm{CaF}_{2}$ (111) surface at room temperature [24]. Simultaneous manipulation of several molecules at room temperature has been performed by Kawai et al [25] as well as in our group [22]. In contrast to the latter studies, here we present the controlled switching of a single molecule at room 
temperature. The manipulation is performed by employing a simple approach, which takes benefit of the $\mathrm{TiO}_{2}(110)$ surface topography, but which is also applicable to other surfaces.

\section{Methods}

The entire experimental work presented in this paper was performed under ultrahigh vacuum conditions (UHV) at room temperature using an NC-AFM operated in the frequency modulation mode [26]. In NC-AFM, a sharp tip mounted at the end of a cantilever is scanned over the surface, while the cantilever is driven at its actual resonance frequency. Because of tip-surface interactions, the resonance frequency changes, which is monitored as a resonance frequency shift $\Delta f$. When imaging in the constant height mode, $\Delta f$ is recorded and displayed as a frequency shift image. When imaging in the constant frequency shift mode, $\Delta f$ is kept constant by regulating the tip-surface distance. For all measurements reported here, the distance feedback loop was set very slow, yielding quasi-constant-height images while still following the overall tilt of the sample surface, i.e. the presented images show $\Delta f$ as a function of the tip position.

The experimental details have been described previously [27]. For all measurements we use n-doped silicon cantilevers (NanoWorld, Neuchâtel, Switzerland) with a resonance frequency of about $300 \mathrm{kHz}$ (type PPP-NCH) operated with an amplitude of about $10 \mathrm{~nm}$. Prior to their use, the cantilevers were $\mathrm{Ar}^{+}$-sputtered at $2 \mathrm{keV}$ for 5 min to remove contaminants. All images are taken with a scanning speed of one line per second and 500 pixels per line. Fast- and slow-scan directions are indicated by the arrows in the upper right corner in each image. The images are displayed such that bright corresponds to a highly attractive interaction while dark corresponds to less attractive or even repulsive interactions.

The molecule used in this study is a 3,4,9,10-perylene tetracarboxylic diimide (PTCDI) derivative, namely $N, N$ /bis(1-hexylheptyl)-PTCDI (CAS no. 110590-84-6) [28, 29]. The chemical structure of this molecule, referred to as PTCDI $\mathrm{S}-13$, is shown in figure 1(a). After a cleaning procedure (heating for $12 \mathrm{~h}$ at $400 \mathrm{~K}$ ) the molecules were sublimated in situ at a temperature of $455 \mathrm{~K}$. For more details on the preparation, adsorption and imaging characteristics see [27].

PTCDI S-13 was studied on the (110) surface of rutile titanium dioxide, which is shown in figure 1(b). The $\mathrm{TiO}_{2}$ samples were crystals of the highest available quality (MTI, Richmond, USA). The unreconstructed (110) surface was prepared by repeated cycles of $\mathrm{Ar}^{+}$sputtering at $1 \mathrm{kV}$ for $15 \mathrm{~min}$ and subsequent annealing at $990 \mathrm{~K}$ for $15 \mathrm{~min}$.

The density functional theory (DFT) calculations were performed with the SIESTA package [30], using ab initio pseudopotentials and the local-density approximation (LDA). More details of the calculations can be found in [27].

For the bare $\mathrm{TiO}_{2}(110)$ surface, several different contrast modes are known [31-34]. In previous investigations we have observed two different imaging modes for the molecules [27], which are shown in figure 2. In mode I (figure 2(a)) the molecules were imaged as bright and unstructured features, while in mode II (figure 2(b)) the molecules were imaged
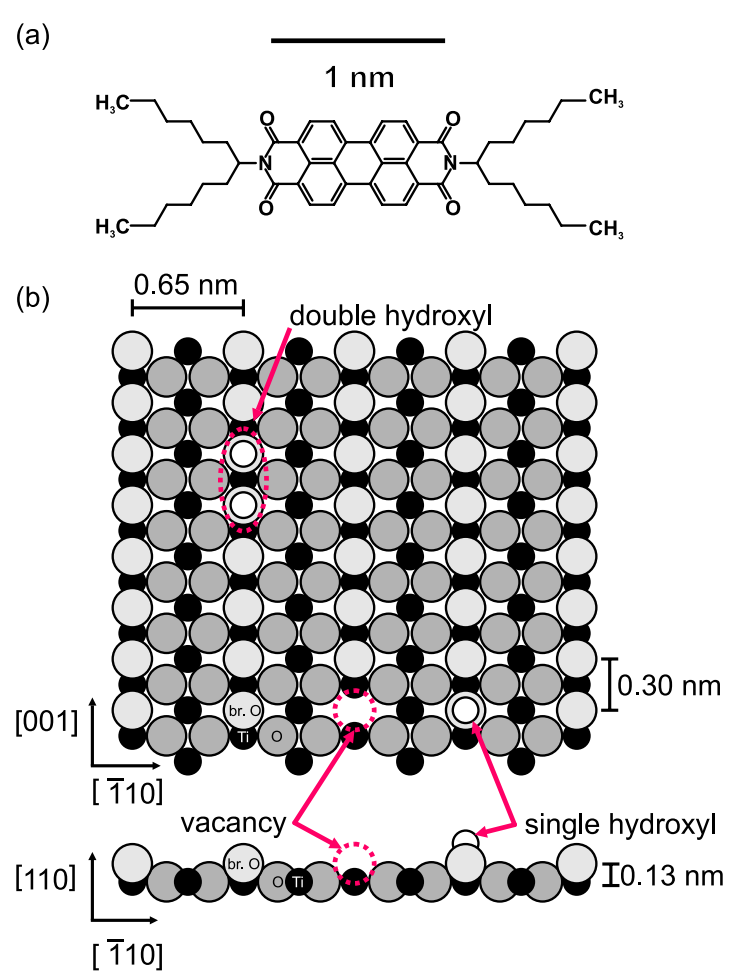

Figure 1. (a) Schematic drawing of an $N, N$ /-bis(1-hexylheptyl)-PTCDI molecule. (b) Schematic drawing of the bulk-truncated rutile $\mathrm{TiO}_{2}(110)$ surface with bridging oxygen rows along the [001] direction. Titanium atoms are shown as small black circles. Oxygen atoms are shown as gray circles; lighter shading represents higher atoms. Defects in the bridging oxygen rows are marked by arrows, showing an oxygen vacancy as well as single and double hydroxyls.

as features with a dark center and a bright rim. The dark center corresponds to a region with less attractive interaction. In mode II, the core of the molecules appears slightly bent (in figure 2(b) two molecules are bent to the left while one molecule is bent to the right), which is related to the adsorption position. As revealed by NC-AFM and STM investigations as well as DFT calculations the molecules adsorb on the bridging oxygen rows. DFT calculations have shown that the molecules adsorb not exactly on top of the oxygen rows but tilted at an angle of about $22^{\circ}$ towards the Ti troughs (see figure 2(c)) [27]. This tilted adsorption position is explaining the previously mentioned bent imaging of the molecules. Molecules bent to the left are adsorbed tilted to the right $\mathrm{Ti}$ troughs and vice versa. When comparing our results to the NC-AFM contrast formation previously reported in the literature [31-34], we can explain the different imaging modes by negative (mode I) and positive (mode II) tip terminations. An explanation limited to electrostatic interactions solely represents a rather simple picture and the tip-sample interaction might involve various other interaction types. In this paper, however, we will use the terms 'positive' and 'negative' for simplicity.

\section{Results and discussion}

In figure 3, a series of three consecutive NC-AFM images is shown, demonstrating the manipulation of a single PTCDI 

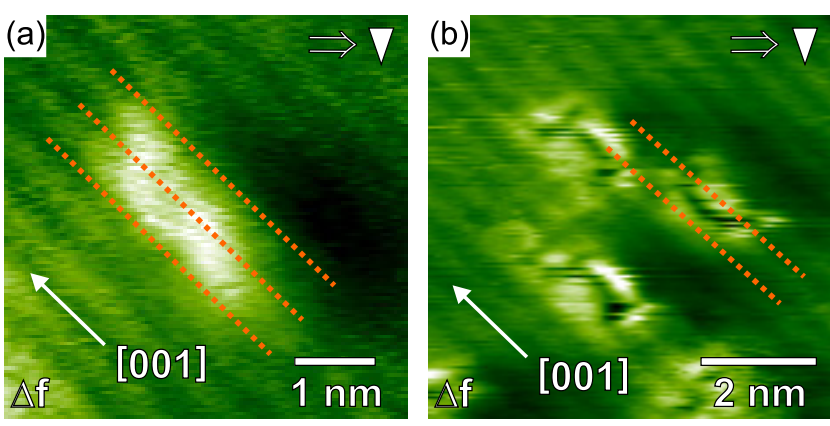

(c)
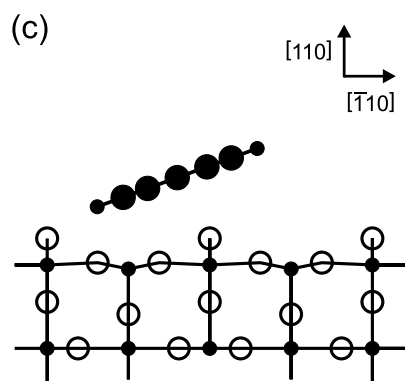

Figure 2. NC-AFM quasi-constant-height images and schematic diagram of the different contrast modes and the adsorption position as revealed by DFT [27]. Image (a) shows a molecule imaged with a negative tip (mode I), while image (b) shows a different sample area with three molecules imaged by a positive-terminated tip (mode II). In each image, the dark substrate rows close to the molecules are highlighted by dotted orange lines, illustrating that the molecule in (a) is centered on a dark row, while the molecules in (b) are centered on a bright row. The schematic drawing in (c) illustrates the energetically favored adsorption position (looking in the [001] direction of the substrate) as revealed by the DFT calculations. Further information can be found in [27].

S-13 molecule. For all images presented, the fast-scan direction is from right to left, while the slow-scan direction switches orientation from image to image. In figure 3(a) three molecules are seen, but we concentrate on the lower two. Due to the molecular appearance (dark center with a bright rim), we can assign the imaging mode to a positively terminated tip (mode II). The upper molecule is bent to the right, while the molecule in the lower part of the image is bent to the left. In (b) an illustration of the different orientations of the molecules on the titania surface is given. We want to stress that the appearance of the molecules does not change as long as the tipsample distance is kept the same. In the following NC-AFM image presented in figure 3(c), the same area is shown again, but now scanned with a higher frequency shift setpoint, i.e. with a higher tip-sample interaction. The higher tip-sample interaction leads to the manipulation of the lower molecule marked by the square. The upper molecule is not manipulated for reasons that are given below. The third NC-AFM image in figure 3(e) again shows the same surface area, but now both molecules are bent to the right, confirming the successful manipulation. In figure 3(d) the area marked by the square from image (c) is reproduced with a larger frame size. The fast scan line, where the manipulation takes place, is marked by red triangles. This backward (scanned from right to left) fast-scan line and the previous forward (left to right) and backward scan lines are given in figure 4 . From figure $4(\mathrm{a})$ it is obvious that the
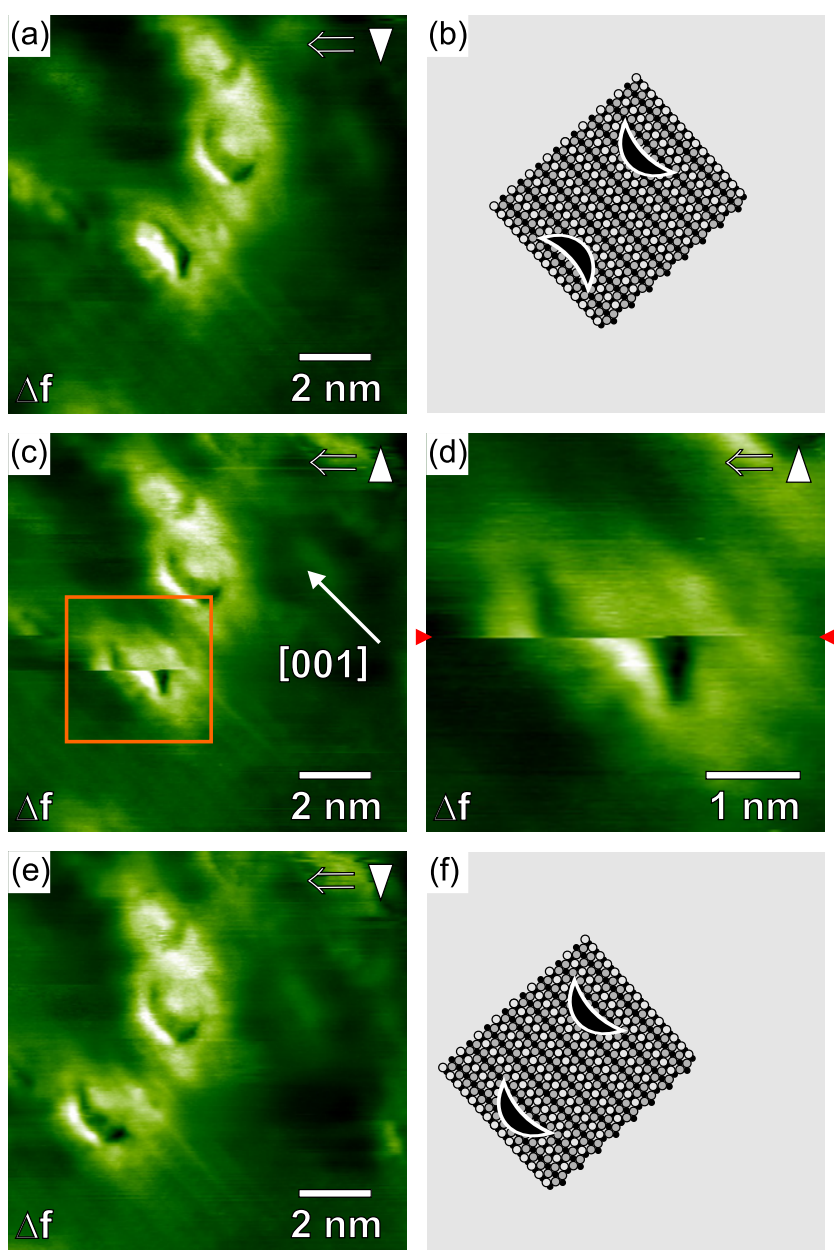

Figure 3. Series of three consecutive quasi-constant-height NC-AFM images showing the manipulation of a single PTCDI S-13 molecule. In (a) two molecules are imaged with a positive-terminated tip (mode II). The upper molecule is bent to the left, while the lower molecule is bent to the right. In (b) a sketch illustrating the two different adsorption geometries is shown. In the following image (c) the $\Delta f$ setpoint is reduced and, consequently, the tip-sample interaction is increased. Due to the higher tip-molecule interaction, the energy barrier for switching the molecule from one side of the bridging oxygen rows to the other is overcome and the lower molecule is manipulated as can be seen by the sharp change in its appearance. Image (d) shows the manipulation process in greater detail. In the following image (e), both molecules are bent to the right, which is also illustrated by the sketch in image (f). The average $\Delta f$ in (a) is $-36.5 \mathrm{~Hz},(\mathrm{c})-37 \mathrm{~Hz}$ and (e) $-37.5 \mathrm{~Hz}$.

absolute value of the frequency shift for the backward scan line is always larger than in the forward scan line, demonstrating that the tip-molecule interaction is higher in the backward scan compared to the forward scan. This effect has its origin in the distance regulation feedback and the tilt of the sample ${ }^{6}$.

6 The left edge of the imaged area is about $0.1 \mathrm{~nm}$ higher than the right edge. Thus, for the forward scan direction the distance feedback has to adjust for a frequency shift with a smaller absolute value by moving the tip closer to the surface. Because of the slow feedback settings, the frequency shift setpoint is not reached completely and, therefore, the measured absolute value of the frequency shift is smaller than for the backward scan line, where the distance feedback has to compensate for too high absolute values of the frequency shift. Thus, the smaller tip-sample interaction in the forward scan direction compared to the backward scan direction is a direct result of the sample tilt and the slow feedback loop settings. 
The backward scan line before the manipulation event is presented in figure 4(b) (line 203 backward, gray squares). Two distinct maxima are visible. The left feature is higher, which has its origin in the tilted adsorption position of the molecule and the sample tilt. The manipulation process takes place in the following backward scan line (line 204 backward, red dots). The manipulation event is marked by the rectangle. Following the way of the tip in this scan line, we start on the right-hand side, where the tip images the first bright rim of the molecule. While the tip is over the dark imaged region of the molecule, the manipulation takes place, which is visible as a steep rise of the absolute value of the frequency shift. After the manipulation, the characteristic pattern of the molecule is imaged again. We now discuss whether the observed manipulation arises due to an attractive pulling or a repulsive pushing type of manipulation. First, we note that the manipulation does not take place at the site of highest tipmolecule attraction (largest absolute value of the frequency shift) but, instead, in the less attractive center of the molecule. Considering the symmetric shape of the molecule, a symmetric tip $^{7}$ placed above the center of the molecule exerts an identical force to the left and to the right part of the molecule. Thus, the lateral attractive force components in the center of the molecule should compensate each other. In fact, due to the slightly tilted adsorption position, the left side is imaged as even more bright than the right side, indicating a stronger attractive interaction of the left side of the molecule towards the tip, which would result in an attractive force pointing towards the right, a direction opposite to the manipulation direction. Hence, a manipulation to the left side due to attractive forces acting between the tip and molecule appears unlikely. This line of argument suggests a repulsive, pushing type of manipulation. As a repulsive interaction is associated with small negative or even positive frequency shifts [35], one would expect a spike in the manipulation curve in figure 4(b) towards small absolute values of the frequency shift. The absence of this spike might be explained by the fast time scale of the manipulation process compared to the NC-AFM data collection.

The higher tip-sample interaction during the backward scan and the different arrangements of the molecules also explain why the lower molecule is switched, while the upper one remains in its original position: while the lower molecule is tilted to the right, the upper molecule is tilted to the left. Considering now a pushing type of manipulation, the upper molecule bent to the right already lies on the left side of the bridging oxygen atoms of the titania surface and, thus, cannot be manipulated in the backward scan.

In figure 4(c), an illustration of the manipulation process is shown. To simplify the drawing the alkyl chains of the PTCDI $\mathrm{S}-13$ molecules are omitted. The molecule is pushed by the NC-AFM tip from the right side to the left side of the bridging oxygen rows. In total, we were able to manipulate around 20 molecules by increasing the tip-molecule interaction. Note that all switching events were exclusively observed when the tip was scanned over the molecule, in other words, we did not observe spontaneous switching events.

${ }^{7}$ Note that the experiment was repeated with different tips, greatly reducing the influence of special tip conditions such as an asymmetric tip shape.
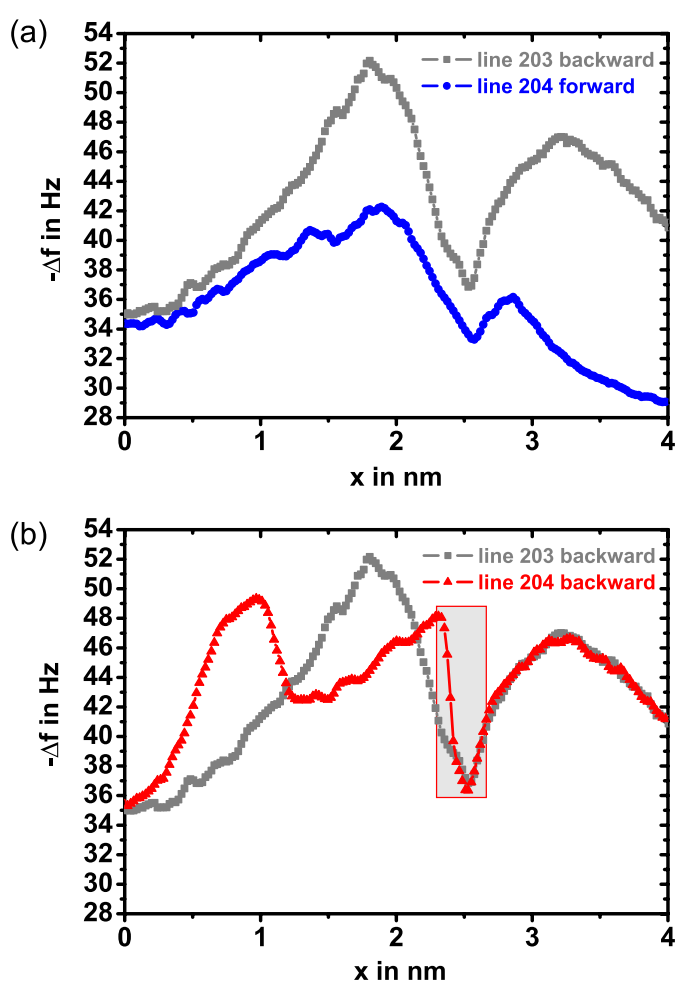

(c)

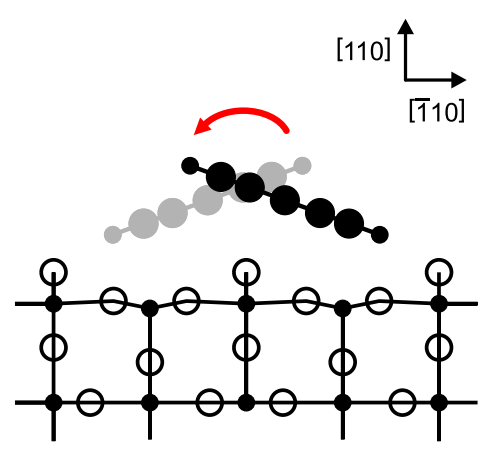

Figure 4. Part (a) shows a forward (blue) and the preceding backward (gray) scan line, revealing a much lower tip-sample interaction in the forward scanning direction. In graph (b) the backward scan line marked by two triangles from figure 3(d) and the preceding backward scan line are shown. Sketch (c) shows a model of the manipulation process. For simplicity the alkyl side chains are not drawn.

From the calculations carried out to elucidate the detailed adsorption position of PTCDI on the rutile (110) surface [27] we are also able to obtain an estimate for the energy barrier, which must be overcome to push the molecule from one side of the bridging oxygen row to the other side. To reduce the needed calculation time, two simplifications are made: first, bare PTCDI without the alkyl chain side groups are considered for the calculations. Second, a full geometry optimization for the whole system (titania substrate plus the molecule) is not performed. This reduces the number of possible variables for the position of the PTCDI molecule to four variables: $X$, the position of the center of the PTCDI in the [110] direction, $Y$, the position in the [001] direction, $Z$, the height of the molecule over the substrate and $\Phi$, the tilt angle of the PTCDI molecule around its long axis. These four 


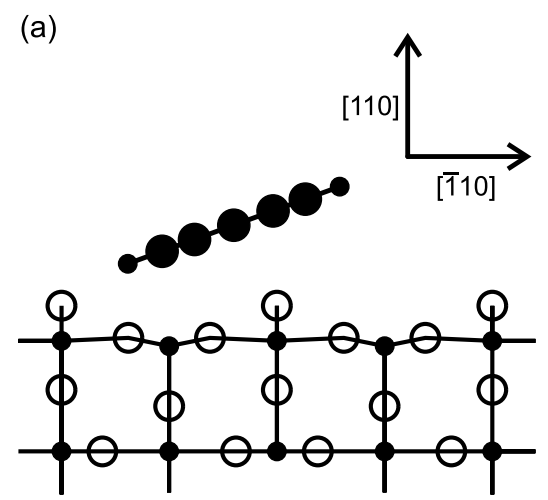

(c)

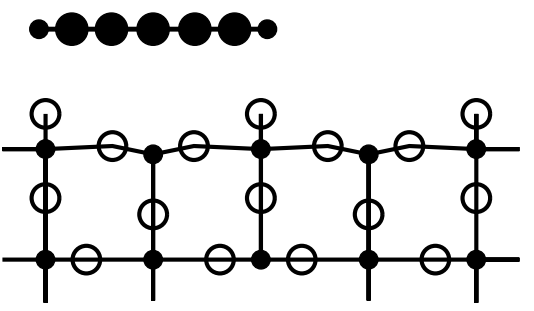

(b)

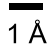

000000

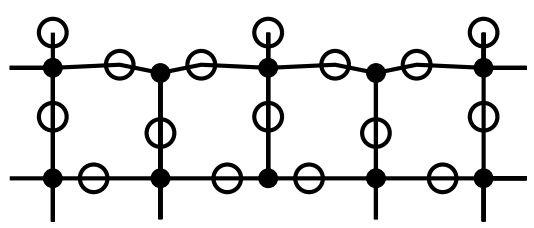

(d)

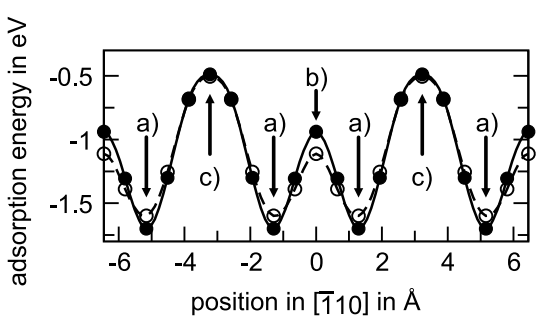

Figure 5. Calculated adsorption structures for PTCDI on the $\mathrm{TiO}_{2}$ surface (adapted from [27]). (a)-(c) show the cross section of the surface looking along the [001] direction with different positions of the molecules. (d) shows the corresponding adsorption energies in dependence on the adsorption positions shown (filled and open circles correspond to $\mathrm{Y}$ coinciding with a titanium trough or a bridging oxygen atom, respectively).

variables are varied and the DFT total energy of the system is calculated for each configuration. Thereafter, the energy is optimized with respect to the parameters by polynomial approximation and least-squares fitting. While this procedure (i.e. neglecting structural deformation of the substrate and molecule upon adsorption) may not be accurate enough to draw final conclusions on precise adsorption energies and details of the adsorption geometry, it allows us to focus on adsorption sites and orientations from a more general point of view. Full geometry optimization has been shown to be extremely slow and, for a system of such a complex nature as the present one, might be subject to being trapped in side minima, leading to wrong conclusions concerning the adsorption site.

For the above-described manipulation event, three different configurations are presented in figures 5(a)-(c), while the corresponding adsorption energies are shown in figure 5(d). Note that two positions along the [001] direction were considered. The filled circles in figure 5(d) represent the calculated data for $\mathrm{Y}$ coinciding with the center of a titanium trough atom, while the open circles show the data for $Y$ coinciding with the center of a bridging oxygen atom.

In part (a), one out of two optimum adsorption positions is shown. Due to the mirror symmetrical configuration, the adsorption energy of the molecule lying on the left of the bridging oxygen row is equal to the adsorption energy of the molecule lying to the right of the bridging oxygen row. The total adsorption energy in this configuration is around $-1.7 \mathrm{eV}$, as seen in part (d). To switch between these two configurations the molecule has to traverse the bridging oxygen row (configuration shown in (b)). This configuration results in a total adsorption energy of nearly $-0.9 \mathrm{eV}$, which is about $0.8 \mathrm{eV}$ higher than the optimal adsorption energy. The resulting energy barrier is very close to the thermal energy of a single molecule at room temperature [36]. However, we do not observe any spontaneous switching of the molecules, indicating that spontaneous switching is not possible. An explanation for this discrepancy might be that the molecules adsorb to the defects of the titania surface (shown in [27]). Other possible explanations include the presence of the alkyl chain side groups of the molecules (not included in the calculations) and also the errors and simplifications of the calculations. In part (c) of figure 5, the energetically most unfavorable configuration with an adsorption energy of around $-0.5 \mathrm{eV}$ is shown, where the molecule lies centered on the titanium atoms in the trough. The difference between this configuration and the optimal adsorption position is around $1.2 \mathrm{eV}$, which is far too high to be overcome by a molecule at room temperature.

As a result of the limited number of different adsorption positions we do not determine the exact height of the energy barriers. In fact, the stated numbers represent a lower estimate of the relevant barriers. Considering the number of simplifications made for the DFT calculations, the experimental observations are in good agreement with the theoretical results.

\section{Summary and conclusion}

In summary, the controlled manipulation of a single molecule is presented using NC-AFM at room temperature. Employing 
a simple approach, a single PTCDI S-13 molecule is switched from lying on one side of the bridging oxygen row of a titania (110) surface to the other side. Careful analysis of the manipulation event suggests the manipulation to be performed in the repulsive pushing mode. The adsorption energies of the relevant adsorption geometries are calculated using DFT and are in very good agreement with the experimental observations. With this study the capability of NC-AFM for single-molecule manipulation at room temperature is demonstrated.

\section{Acknowledgments}

Stimulating discussions with Michael Reichling are gratefully acknowledged. This work has been supported by the German Research Foundation (DFG) through the Emmy Noetherprogram (KU1980/1-2) and the Niedersachsen PhD program 'Synthesis and Characterisation of Surfaces and Interfaces assembled from Clusters and Molecules'.

\section{References}

[1] Eigler D M and Schweizer E K 1990 Nature 344524

[2] Otero R, Rosei F and Besenbacher F 2006 Annu. Rev. Phys. Chem. 57497

[3] Grill L 2008 J. Phys.: Condens. Matter 20053001

[4] Parschau M, Hug H J, Rieder K-H and Ernst K-H 2010 Surf. Interface Anal. 421629

[5] Orisaka S, Minobe T, Uchihashi T, Sugawara Y and Morita S 1999 Appl. Surf. Sci. 140243

[6] Giessibl F J 1995 Science 26768

[7] Reichling M and Barth C 1999 Phys. Rev. Lett. 83768

[8] Nimmrich M, Kittelmann M, Rahe P, Mayne A J, Dujardin G, von Schmidsfeld A, Reichling M, Harneit W and Kühnle A 2010 Phys. Rev. B 81 201403(R)

[9] Nishi R, Miyagawa D, Seino Y, Yi I and Morita S 2006 Nanotechnology 17 S142

[10] Sugimoto Y, Jelinek P, Pou P, Abe M, Morita S, Perez R and Custance O 2007 Phys. Rev. Lett. 98106104

[11] Ternes M, Lutz C P, Hirjibehendin C F, Giessibl F J and Heinrich A J 2008 Science 3191066

[12] Sugimoto Y, Pou P, Custance O, Jelinek P, Abe M, Perez R and Morita S 2008 Science 322413

[13] Custance O, Perez R and Morita S 2009 Nat. Nanotechnol. 4803
[14] Watkins M B and Shluger A L 2006 Phys. Rev. B 73245435

[15] Trevethan T, Watkins M, Kantorovich L N and Shluger A L 2007 Phys. Rev. Lett. 98028101

[16] Trevethan T, Kantorovich L, Polesel-Maris J, Gauthier S and Shluger A 2007 Phys. Rev. B 76085414

[17] Dieška P and Štich I 2007 Nanotechnology 18084016

[18] Atodiresei N, Caciuc V, Blügel S and Hölscher H 2008 Phys. Rev. B 77153408

[19] Martsinovich N, Kantorovich L, Fawcett R H J, Humphry M J and Beton P H 2008 Small 4765

[20] Martsinovich N and Kantorovich L 2009 Nanotechnology 20135706

[21] Kunstmann T, Schlarb A, Fendrich M, Wagner T and Möller R 2005 Phys. Rev. B 71 121403(R)

[22] Loske F and Kühnle A 2009 Appl. Phys. Lett. 95043110

[23] Rahe P, Nimmrich M, Greuling A, Schütte J, Stará I G, Rybáček J, Huerta-Angeles G, Starý I, Rohlfing M and Kühnle A 2010 J. Phys. Chem. C 1141547

[24] Hirth S, Ostendorf F and Reichling M 2006 Nanotechnology 17 S148

[25] Kawai S, Maier S, Glatzel T, Koch S, Such B, Zimmerli L, Fendt L-A, Diederich E and Meyer E 2009 Appl. Phys. Lett. 95103109

[26] Albrecht T R, Grütter P, Horne D and Rugar D 1991 J. Appl. Phys. 69668

[27] Schütte J, Bechstein R, Rahe P, Rohlfing M, Kühnle A and Langhals H 2009 Phys. Rev. B 79045428

[28] Demmig S and Langhals H 1988 Chem. Berichte-Recueil 121225

[29] Langhals H 2005 Helv. Chim. Acta 881309

[30] Soler J M, Artacho E, Gale J D, García A, Junquera J, Ordejón P and Sánchez-Portal D 2002 J. Phys.: Condens. Matter 142745

[31] Lauritsen J V, Foster A S, Olesen G H, Christensen M C, Kühnle A, Helveg S, Rostrup-Nielsen J R, Clausen B S, Reichling $\mathrm{M}$ and Besenbacher F 2006 Nanotechnology 173436

[32] Enevoldsen G H, Foster A S, Christensen M C, Lauritsen J V and Besenbacher F 2007 Phys. Rev. B 76205415

[33] Bechstein R, González C, Schütte J, Jelínek P, Pérez R and Kühnle A 2009 Nanotechnology 20505703

[34] Yurtsever A, Sugimoto Y, Abe M and Morita S 2010 Nanotechnology 21165702

[35] Rahe P, Bechstein R, Schütte J, Ostendorf F and Kühnle A 2008 Phys. Rev. B 77195410

[36] Schütte J, Bechstein R, Rohlfing M, Reichling M and Kühnle A 2009 Phys. Rev. B 80205421 